\title{
ALK rearrangement: a high-frequency alteration in ovarian metastasis from lung adenocarcinoma
}

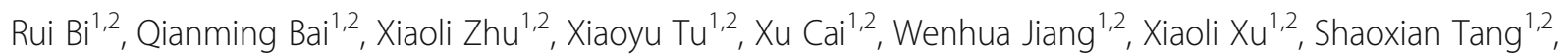
Huijuan Ge ${ }^{1,2}$, Bin Chang ${ }^{1,2}$, Yufan Cheng ${ }^{1,2}$, Hualei Gan ${ }^{1,2}$, Xiaoyan Zhou ${ }^{1,2}$ and Wentao Yang ${ }^{1,2^{*}}$

\begin{abstract}
Background: Ovarian metastatic tumors from lung adenocarcinoma are rare, and a serial study of these tumors is lacking to date. Additionally, a better understanding of the clinicopathological and molecular characteristics of metastatic tumors is needed.

Methods: Seven cases of ovarian metastasis from lung adenocarcinoma from 2013 to 2017 at our institute were investigated. The results were combined with those found in literature review. A total of 16 cases were analyzed in the present study. We examined clinicopathological and immunohistochemical characteristics, further detected ALK rearrangement by FISH (fluorescence in situ hybridization), and assessed EGFR and KRAS mutations using Sanger sequencing or the amplification refractory mutation system (ARMS).

Results: The mean age of the patients was 44.6 years (range, 33-56 years). Eleven of sixteen patients developed ovarian tumors within a mean time of 18.5 months (range, 5-48 months) from the initial diagnosis of lung adenocarcinoma; 5 patients had lung tumors and ovarian masses simultaneously. Five tumors (5/16, 31\%) occurred in the bilateral ovaries, and the others were unilateral ovarian tumors $(11 / 16,69 \%)$. All seven cases from our institute were positive for TTF-1 and Napsin A but negative for PAX8. In four cases, ALK (D5F3) was diffusely and strongly expressed, with ALK rearrangements (4/7,57\%). Overall, ALK rearrangement was found by FISH or immunohistochemistry in 11/16 (69\%) cases. In two cases, EGFR mutations in exons 19 and 21, respectively, were found. One patient did not detected EGFR or ALK mutation in the metastatic tumor, but the primary lung adenocarcinoma did harbor an EGFR mutation. Two cases had no alterations in three genes above. Although the mean survival time of the patients with ALK rearrangement was longer than those without (mean survival time 25 $\mathrm{m}$ vs. $20 \mathrm{~m}$ ), no statistical significance of the difference was found.

Conclusions: As the largest case series of ovarian metastasis from lung adenocarcinoma, our findings indicate that ALK rearrangement is the most common molecular alteration. Although patients with ALK rearrangement appear to have a better prognosis than do those without ALK rearrangement, more cases with longer follow-up and multivariant analysis are needed to clarify this point.
\end{abstract}

Keywords: Ovary, Metastatic lung adenocarcinoma, ALK rearrangement

\footnotetext{
* Correspondence: yangwt2000@163.com

'Department of Pathology, Fudan University Shanghai Cancer Center, Fudan University, 270 Dong An Road, Shanghai 200032, China

${ }^{2}$ Department of Oncology, Shanghai Medical College, Fudan University, 270

Dong An Road, Shanghai 200032, China
}

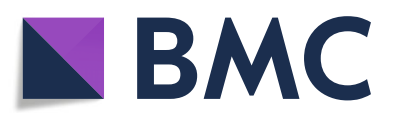

(c) The Author(s). 2019 Open Access This article is distributed under the terms of the Creative Commons Attribution 4.0 International License (http://creativecommons.org/licenses/by/4.0/), which permits unrestricted use, distribution, and reproduction in any medium, provided you give appropriate credit to the original author(s) and the source, provide a link to the Creative Commons license, and indicate if changes were made. The Creative Commons Public Domain Dedication waiver (http://creativecommons.org/publicdomain/zero/1.0/) applies to the data made available in this article, unless otherwise stated. 


\section{Background}

Lung cancer is the second most common cancer, and most cases are diagnosed at advanced stages. The 5-year survival rate of lung cancer is less than $20 \%$. Lung cancer in non-smokers appears to be a distinct disease caused by driver mutations that are different from the genetic changes observed in lung cancer in smokers. In western countries, rearrangements in the gene $(A L K)$ encoding anaplastic lymphoma receptor tyrosine kinase have been found in only $3-7 \%$ of lung adenocarcinomas; in contrast, EGFR mutation is approximately $10-15 \%$, more frequent than $A L K$ rearrangement $[1,2]$. Nevertheless, these two alterations appear to be mutually exclusive in patients [3, 4], and only recent rare cases have shown concomitant alterations in $A L K$ and EGFR [5, 6].

Ovarian metastatic tumors derive from many sites. The common primary sites include gastrointestinal tract, pancreas, and gynecologic tract (the cervix and uterus) [7]. However, lung carcinoma metastasis to the ovary is rarely reported. Reports of these metastatic tumors have included all major lung cancer histotypes, with small cell carcinomas comprising the largest proportion, followed by adenocarcinomas, large cell carcinomas and squamous cell carcinomas [8]. As stated above, lung adenocarcinoma metastases to the ovary are rare, and the incidence is not yet clear. Lung metastasis to uncommon sites occurs in less than $5 \%$ of cases, with metastatic adenocarcinoma to the ovary occurring in only $0.07 \%$. So the frequency of total lung tumor metastasis to the ovary is estimated to be less than $0.01 \%$ [9]. More remarkably, although ovarian metastasis from lung carcinoma has an extremely low incidence, $A L K$ rearrangement in these patients has occasionally been reported [10-17].

In the present study, we investigated 7 consecutive cases of lung adenocarcinoma metastasis to the ovary at our institute with cases-review in the literature. We obtained clinicopathological data regarding major molecular alterations for targeted therapy. To the best of our knowledge, this study is the largest panel of ovarian metastatic tumors from lung adenocarcinomas focusing on genetic alterations to date.

\section{Methods}

\section{Samples}

Seven cases of lung carcinoma metastasis to the ovary, including 5 treated with surgery at Fudan Cancer Center and 2 consultation cases, were reviewed in the Department of Pathology of Fudan University Shanghai Cancer Center in 2013-2017. Clinical information and gross features were obtained from medical records. Formalinfixed, paraffin-embedded tissue blocks or unstained slides were reprocessed for hematoxylin and eosin staining, immunohistochemistry and molecular analysis. All cases were reviewed by two senior pathologists who verified the diagnosis. The present study was approved by our institutional ethics committee. We also reviewed unselected reported cases of ovarian metastasis from lung adenocarcinoma in the English literature in PubMed, and 9 cases with molecular alterations were retrieved. In total, 16 cases were analyzed in the present study.

\section{Immunohistochemistry}

Immunohistochemistry analyses, including detection of PAX8, ER, PR, TTF-1, Napsin A, ALK (D5F3), CK7, CK20, CDX2 and HNF-1 $\beta$, were performed for all 7 cases using a Ventana Benchmark XT autostainer (Ventana Medical Systems Inc., Tucson, AZ, USA). Appropriate positive and negative controls were included. A list of antibodies used in this study is shown in Table 1.

\section{EGFR and KRAS mutational analysis}

The mutational statuses of EGFR (exons 18, 19, 20 and 21 ) and KRAS (exon 2) were determined by polymerase chain reaction (PCR)-based direct gene sequencing, as previously described [18], or by the amplification refractory mutation system (ARMS). In brief, genomic DNA from the 7 tumors was extracted using a QIAamp DNA FFPE Tissue Kit (Qiagen, Valencia, CA, USA) according to the manufacturer's instructions. The primers used for EGFR and KRAS are listed in Table 2. The PCR products were confirmed by agarose gel electrophoresis, purified using a DNA Clean/Extraction Kit (GeneMarkBio, A\&D Technology, Beijing, China), and submitted for direct sequencing using a BigDye Terminator Cycle Sequencing Kit (Applied Biosystems) according to the manufacturer's protocol. The sequencing products were ethanol precipitated before being assessed using a 3500 Genetic Analyzer (Applied Biosystems), and the resulting sequence data were analyzed using Chromas software. Each mutation was verified in both the sense and antisense directions and was independently evaluated by two

Table 1 Antibodies used for immunohistochemistry

\begin{tabular}{llll}
\hline Antibody & Dilution & Clone & Manufacturer \\
\hline TTF-1 & Ready-use & SPT24 & Leica \\
NapsinA & $1: 500$ & KCG1.1 & Abcam \\
ALK & Ready-use & D5F3 & Ventana \\
PAX8 & $1: 150$ & MRQ-50 & Cell Marque \\
ER & Ready-use & SP1 & Ventana \\
PR & Ready-use & 1E2 & Ventana \\
CK7 & $1: 100$ & SP52 & Ventana \\
CK20 & Ready-use & SP33 & Ventana \\
CDX2 & $1: 50$ & EPR2764Y & Maxim \\
HNF-13 & $1: 500$ & polyclone & Sigma \\
P63 & Ready-use & 4A4 & Roche \\
P40 & Ready-use & ER8 & Maxim \\
\hline
\end{tabular}


Table 2 The primers of EGFR and KRAS on hot spots

\begin{tabular}{ll}
\hline EGFR & \\
\hline Exon 18 & F: 5'-AGCATGGTGAGGGCTGAGGTGAC-3' \\
R: 5'-ATATACAGCTTGCAAGGACTCTGG-3' \\
Fxon 19 & F: 5'-CCAGATCACTGGGCAGCATGTGGCACC-3' \\
R: 5'-AGCAGGGTCTAGAGCAGAGCAGCTGCC-3 & F: 5'-GATCGCATTCATGCGTCTTCACC-3' \\
& R: 5'- TTGCTATCCCAGGAGCGCAGACC-3 \\
Exon 21 & F: 5'-TCAGAGCCTGGCATGAACATGACCCTG-3' \\
KRAS & R: 5'- GGTCCCTGGTGTCAGGAAAATGCTGG-3' \\
Exon 2 & F: 5'-AGGCCTGCTGAAAATGACTG-3' \\
& R: 5'-TCAAAGAATGGTCCTGCACC-3' \\
\hline
\end{tabular}

investigators. ARMS was carried out according to the manufacturer's instructions (AmoyDx of Xiamen, Fujian Province, China).

\section{$A L K$ rearrangement based on FISH}

Vysis $A L K$ Break Apart FISH Probe Kit (Abbott Molecular) was used for $A L K$ testing, as described previously [19]. Fifty or more non-overlapping nuclei were counted by two professional molecular pathologists according to standard criteria in our laboratory. The positive cut-off for $A L K$ rearrangement was at least $15 \%$ tumor cells with a split pattern and/or single orange signal without a corresponding green signal [20]. An increased number of nuclei or alterative nuclei were counted if the results were close to the cut-off values.

\section{Results}

\section{Clinicopathological data}

Among the seven cases in our cohort and the nine cases previously reported, patient ages ranged from 33 to 56 years (mean 44.6 years). Among 12 patients for which smoking history was known, 1 patient was a light smoker (2.5-pack years), 1 patient smoked 1-pack per day, and the other patients had no smoking history (10/12, 83\%). In 5 cases $(5 / 16,31 \%)$, the lung adenocarcinoma and ovarian metastatic tumors occurred simultaneously. For the remaining 11 cases $(11 / 16,69 \%)$, ovarian tumors were observed at 5-48 months (mean 18.5 months) following the diagnosis of the lung adenocarcinoma. Computed tomography revealed bilateral ovarian tumors in only five patients $(5 / 16,31 \%)$; the others presented unilateral tumors $(11 / 16,69 \%)$. Concurrent metastatic sites were present in most cases $(13 / 16,81 \%)$, including the bone (6 cases), brain ( 5 cases), supraclavicular lymph node (3 cases), liver ( 2 cases), and pleural lymph node (1 case) but were absent in 3 cases $(3 / 16,19 \%)$ (Table 3$)$. Serum CA125 was elevated with median values in the range of $100-500 \mathrm{U} / \mathrm{ml}$ in 5 recorded cases.

\section{Pathological features}

On gross examination, the ovarian tumors exhibited smooth outer surface. Macroscopic omental cake was present in one case (LTO_2). The mean size of 13 ovarian tumors with available data was $11 \mathrm{~cm}$ (range, 4-21.5 $\mathrm{cm})$. The cut surface was predominantly solid in 7 cases, whereas it was cystic in two cases; the other 4 cases included mixed solid and cystic surfaces. The solid area of the tumor had a medium firmness level (LTO_1, Fig. 1). Large cavities in two of the tumors (LTO_3 and 7) were filled with dark brown viscous liquid and showed partial papillary architecture. The clinical and macroscopic features of all cases are summarized in Table 3.

Upon microscopic observation, all 16 cases were found to be adenocarcinomas, including 5 cases with solid or prominent solid features, 8 cases with prominent acinar features (Fig. 2a-d) and 2 cases with a mixed solid and acinar pattern. One case did not represented the histological subtype in the article. LTO_1 was characterized by solid features, and the tumor cells had distinct cell membrane and little mucin, similar to squamous cell carcinoma, but were negative for $\mathrm{p} 63$ and $\mathrm{p} 40$. Prominent solid patterns and sheets of tumor cells were also observed in other four cases, presenting polymorphic epithelioid cells with eosinophilic or clear cytoplasm and unclear cell borders. Remarkably, signet ring cells were observed in 3 cases. Tumor cells with an eosinophilic cytoplasm were attached to the cystic wall and exhibited hemorrhage and inflammatory infiltration, with a mixed acinar and solid growth pattern in LTO_3. The acinar features included long tubules but with little mucus in the lumen in 8 cases. Signet ring cells were observed in LTO_16. Additionally, acinar features with micropapillaries in acinar cells were found in LTO_5, and eosinophilic, mucinous fluid was observed in some acinar cells. In LTO_7, prominent mucin were present in acinar cells, which showed a cribriform pattern. In the present case series, the acinar pattern was slightly more common than solid pattern. However, $4 / 5$ cases with solid features harbored $A L K$ rearrangement.

\section{Immunohistochemistry}

ER, PR, HNF-1 $\beta$ and PAX8 expression was negative in our 7 cases, while TTF-1, Napsin A and CK7 were expressed in all 7 cases. No cases were positive for CK20 or CDX2. LTO_1 was negative for p63 and p40. Four of 7 cases showed diffuse and strong positivity for ALK (D5F3) (Fig. 2e). Interestingly, the 2 cases with solid or prominent solid features were both ALK positive. The immunohistochemistry results are presented in Table 3.

\section{Genetic alterations in EGFR and KRAS and $A L K$ rearrangement}

In seven cases, sequencing was successful and revealed mutations in EGFR exons 18, 19, 20 and 21 


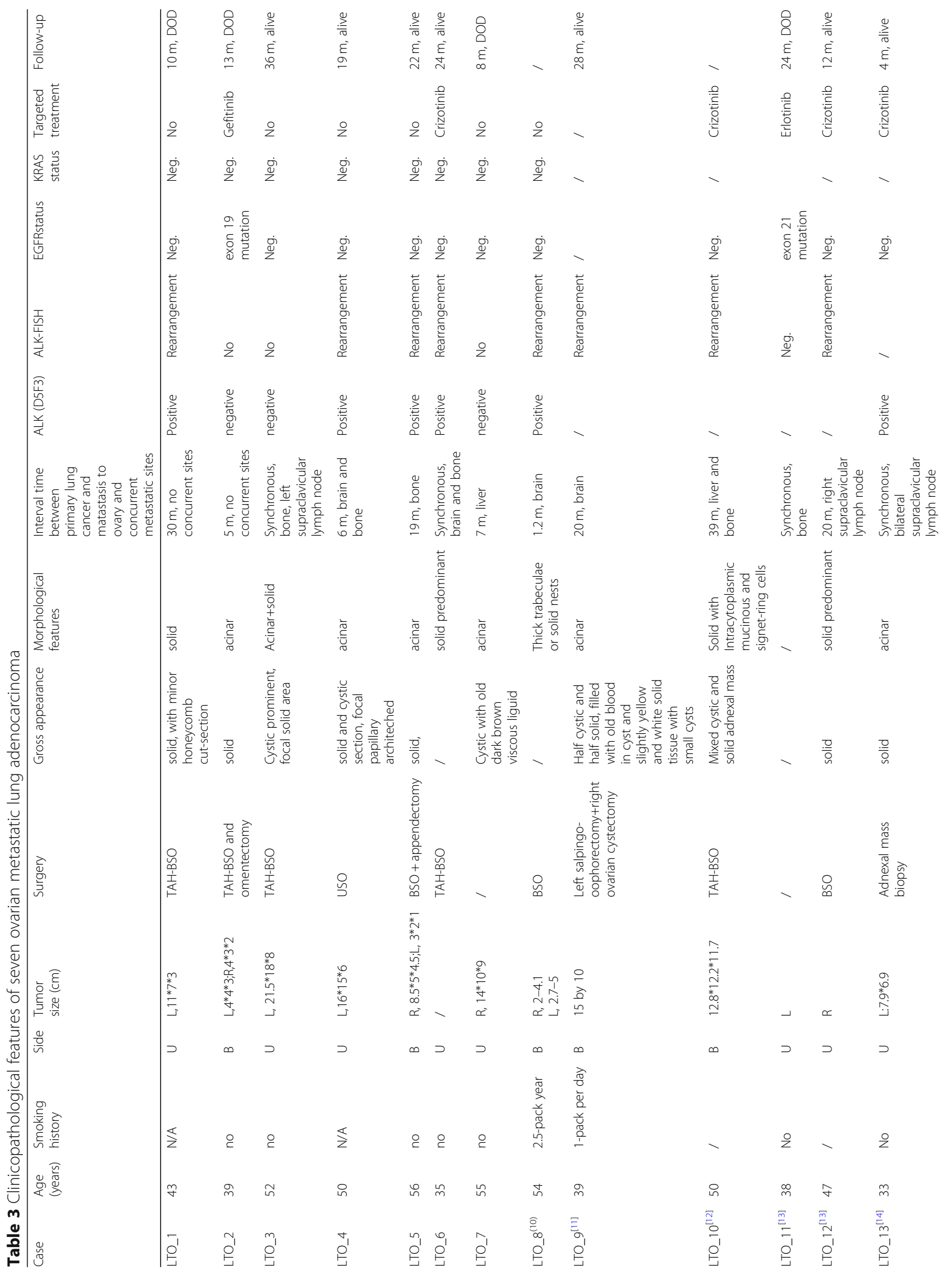




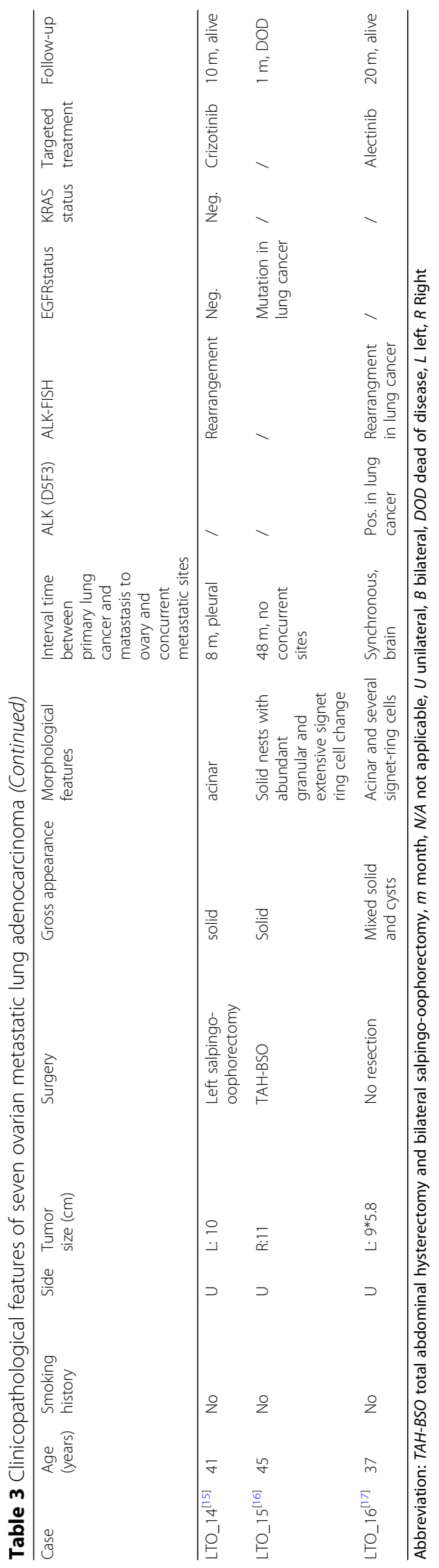




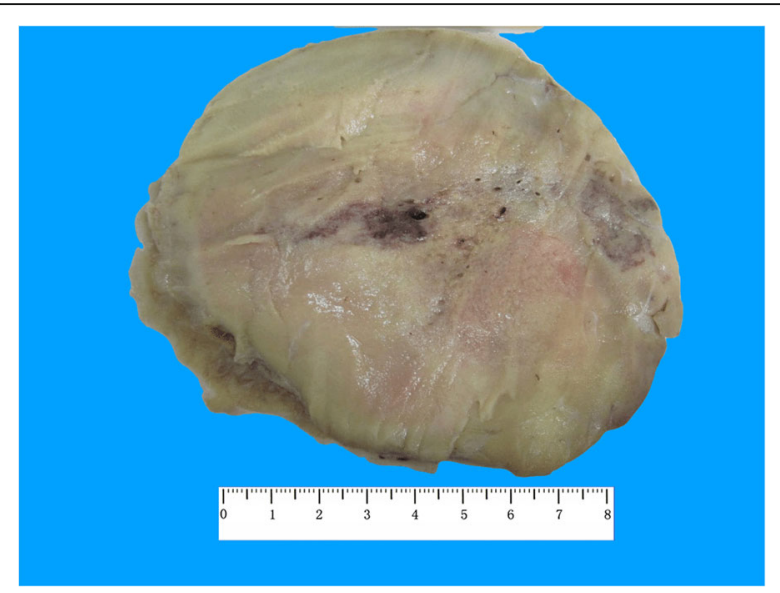

Fig. 1 LTO_1 showed a solid tumor with a grey-yellow cut surface and focal hemorrhage and KRAS exon 2; a frameshift mutation of EGFR exon 19 was detected in one of seven cases (Fig. 3, Case LTO_2). Wild-type EGFR and KRAS genes were found in the remaining 6 cases, though $A L K$ rearrangement was present in 4. Among all 16 cases, FISH or immunohistochemistry revealed an $A L K$ rearrangement in 10. For one patient (LTO_16), molecular examination of the ovarian tumor was not performed, but the lung cancer harbored an $A L K$ rearrangement. Based on the high concordance of $A L K$ status between the primary and metastatic tumors [20], we presumed $A L K$ rearrangement in 11/16 (68.8\%) cases in the present study. Two cases showed EGFR mutation in exon 19 and 21, respectively. One patient did not exhibit an EGFR mutation or $A L K$ rearrangement in the metastatic tumor, but the primary lung adenocarcinoma did carry an EGFR mutation. The remaining 2 cases had no EGFR or KRAS mutation or ALK rearrangement.
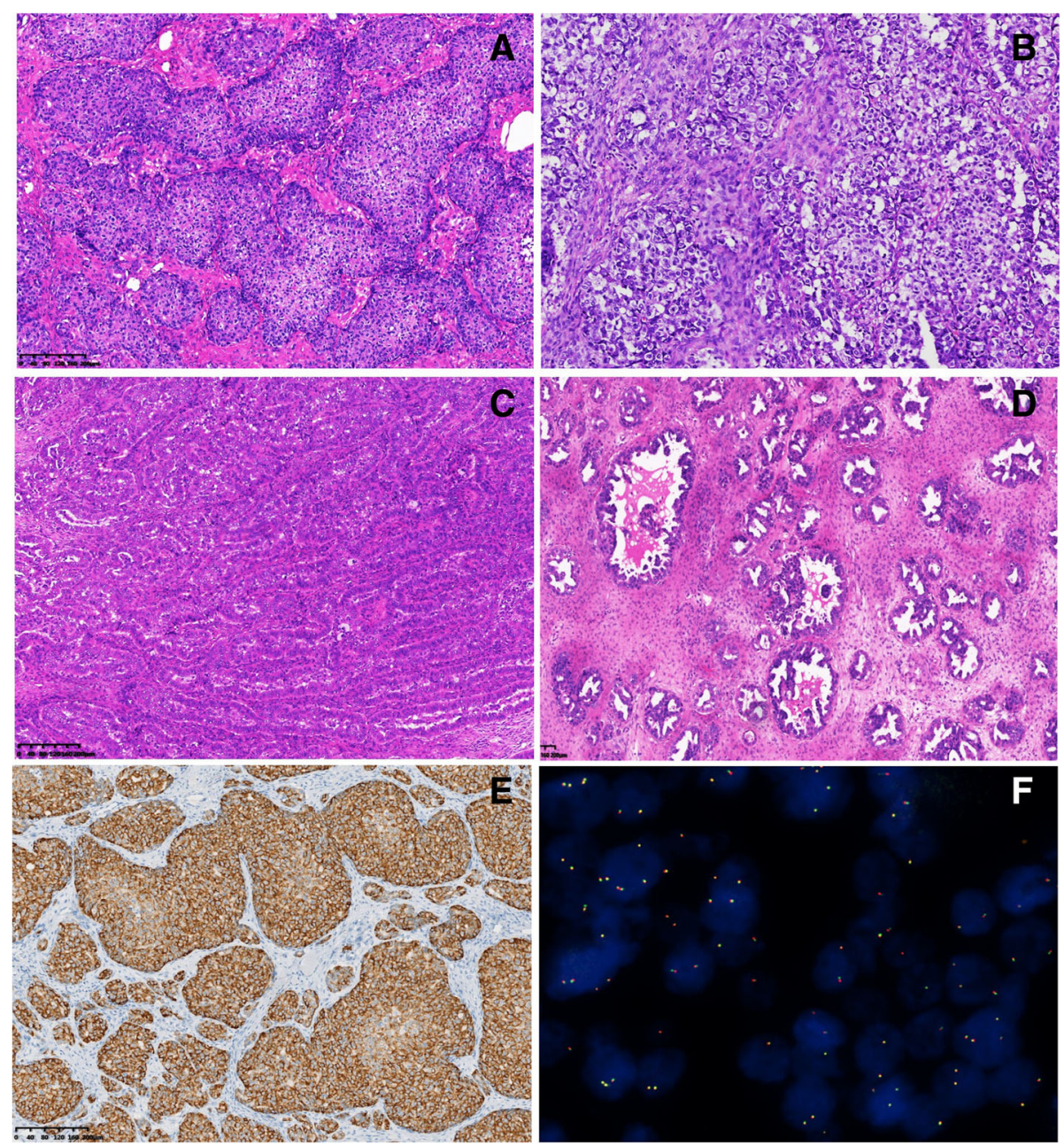

Fig. 2 Histological subtypes of ovarian metastatic lung adenocarcinomas. a and b, LTO_1 and LTO_6, solid subtype; c and d, LTO_4 and LTO_5, acinar subtype; e. ALK (D5F3) diffuse expression in LTO_1; f. ALK rearrangement with split orange and green signals by FISH 


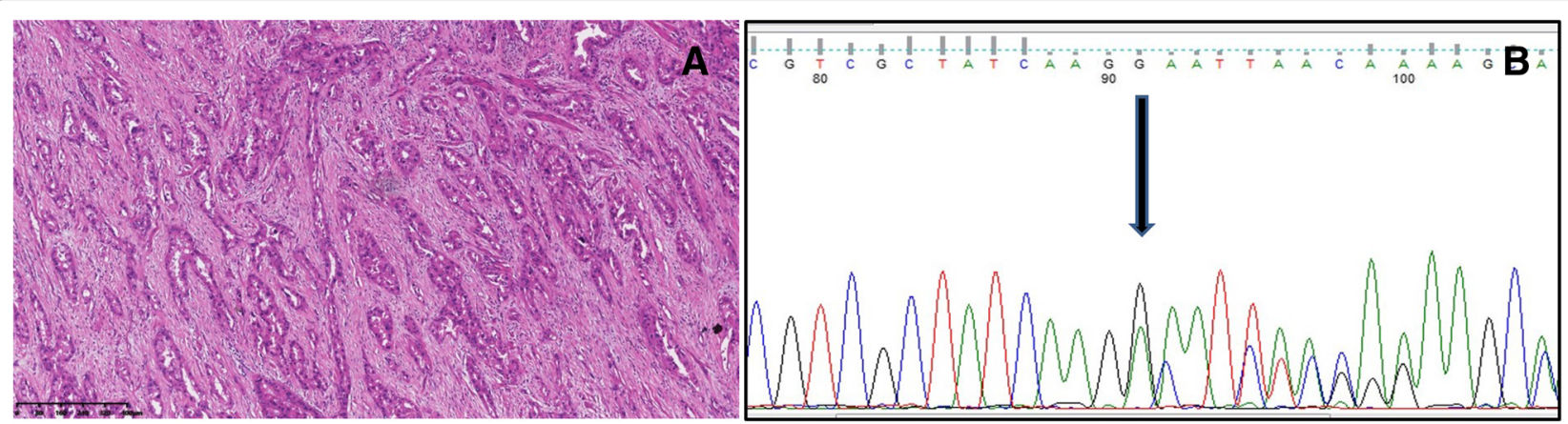

Fig. 3 a. Case LTO_2 showed an acinar subtype. b. EGFR mutation in exon 19, p.746-750del

\section{Follow-up}

The 7 patients in our cohort were followed up until June 7, 2019. The follow-up time of the 9 patients from the English literature were obtained from the original articles. All 16 patients underwent chemotherapy, and 8 received targeted drugs: gefitinib and Tarceva for EGFR mutations and crizotinib and alectinib for $A L K$ rearrangements. Five patients died in 10 months $(\mathrm{m}), 13 \mathrm{~m}$, $8 \mathrm{~m}, 24 \mathrm{~m}$ and $1 \mathrm{~m}$, after the diagnosis of ovarian metastasis from lung adenocarcinoma, including a patient harboring an ALK rearrangement (LTO_1) who did not undergo targeted therapy and had hypothyroidism for 3 years. Two patients, both carrying an EGFR mutation (LTO_2 and LTO_11), were treated with gefitinib or Tarceva. One patient (LTO_15) exhibited an EGFR mutation in the lung tumor but the mutation status of the ovarian metastatic tumor was unknown. The fifth patient who died (LTO_7) exhibited no ALK/EGFR alterations and did not undergo targeted therapy. Of the 9 surviving patients, 8 had $A L K$ rearrangements. Hence, two groups were formed based on the molecular alterations present. The first group included 11 patients with $A L K$ rearrangements. The mean survival of this group was $25 \mathrm{~m}$, with only 1 death from the disease. The other group of 5 patients did not have $A L K$ rearrangements; the mean survival was $20 \mathrm{~m}$, but only 1 patient survived. Although survival was not significantly different between the two groups $(P=0.110)$, longer follow-up with additional samples may clarify the difference in survival between these two groups (Fig. 4). Additional details are listed in Table 3.

\section{Discussion}

The most common non-small cell lung cancer (NSCLC) metastatic sites are the brain [21], followed by the bone and liver [22]. All other organ metastases comprise less than $5 \%$ of cases, and thus, they may be defined as uncommon metastases. The anatomical sites in decreasing order of frequency are the soft tissue, kidney, pancreas, spleen, peritoneum, intestine, bone marrow, eye, ovary, thyroid, heart, breast, tonsil nasal cavity [9], and gastric region [23]. The total frequency of lung metastasis to the ovary is estimated to be less than $0.01 \%$ [9]. Due to the paucity of reported cases, the clinicopathological features of these tumors remain unknown. To the best of our knowledge, this is the largest number of cases of ovarian metastasis from lung adenocarcinoma examined to date. Although the ovary is an uncommon metastatic site in the female reproductive tract, uterine cervix metastasis from lung adenocarcinoma harboring $A L K$ rearrangement has been reported [24], as have concurrent cervix and breast metastases [25]. In our cohort, the minority (19\%) of ovarian metastatic tumors involved only

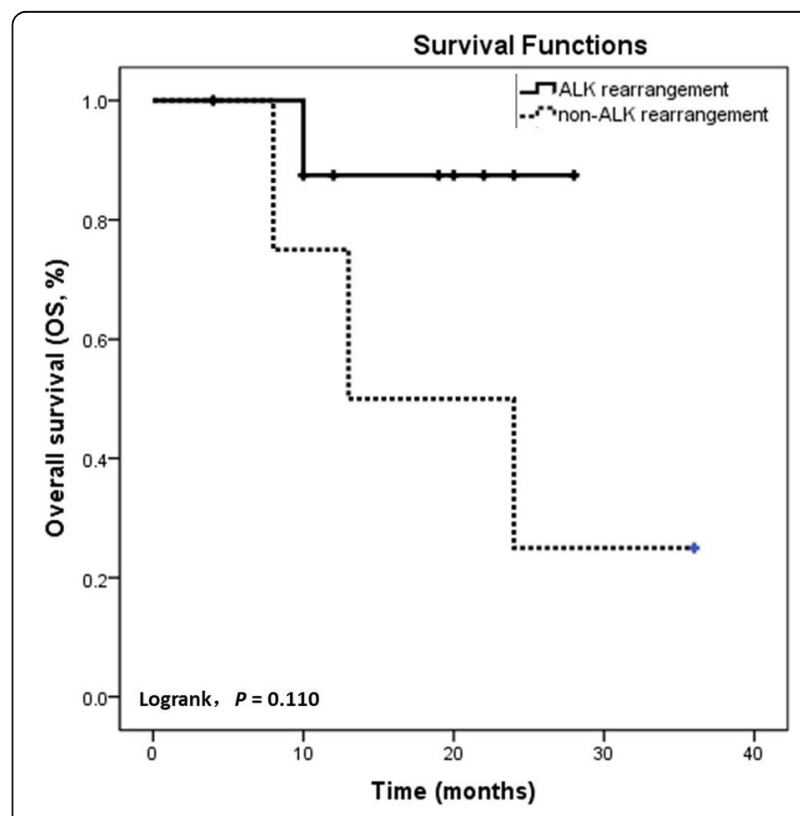

Fig. 4 Kaplan-Meier survival analysis and log-rank analysis of patients with or without $A L K$ rearrangements. Patients with ALK rearrangement had a better prognosis than did those without ALK rearrangement, though no statistically significant difference in survival (log-rank test, $P=0.110$ ) was observed due to the small cohort. One patient who did not undergo targeted therapy for ALK rearrangement died. To some extent, ALK inhibitors are effective for these patients 
one metastatic site, whereas the majority of ovarian metastatic tumors were concurrent with other metastatic sites (81\%), especially the bone and brain.

Ovarian mucinous carcinoma accounts for only $3-4 \%$ of all primary tumors [26], and most cases constitute metastatic tumors from diverse sites. As previously noted by Lee et al. [27], metastatic tumors of the ovary tend to show bilateral masses. However, unilateral masses were common in our cohort of metastatic lung adenocarcinomas $(11 / 16,68.8 \%)$, in contrast to Krukenberg tumors with bilateral masses. This is consistent with unilateral ovarian metastasis in twelve of nineteen cases in Irving's report [8]. Furthermore, tumors greater than $10 \mathrm{~cm}$ in size tended to be primary ovarian tumors, but the mean size in the present study was $11 \mathrm{~cm}$. These findings indicate that ovarian metastatic tumors from lung adenocarcinomas have distinct clinicopathological features compared with those from other organs, and some characteristics may result in misdiagnosis of primary ovarian tumors.

Metastatic mucinous carcinomas mainly include those of the gastrointestinal tract, pancreatic, and gynecologic organs $[27,28]$. Lung adenocarcinoma metastases to the ovary are extremely rare and comprise less than $0.01 \%$ of cases [9]. Although metastatic ovarian tumors prior to the primary lung adenocarcinomas were not found in our cohort, 5 cases $(16 \%)$ of ovarian tumors were reported prior to the lung carcinoma, including 1 adenocarcinoma [8]. Hence, differentiating primary ovarian tumors from metastatic tumors is a major challenge, especially for mucinous ovarian carcinomas. A panel of immunohistochemical markers should be used for differential diagnosis. PAX8 is recognized as a sensitive marker of the gynecologic tract [29], but it was absent in all 7 cases in our cohort. TTF-1 and Napsin A, which are commonly-used pulmonary-origin markers, are helpful in distinguishing these cases from cases of primary ovarian mucinous carcinoma [30]. TTF-1 and Napsin A were expressed in all 7 cases, and metastatic lung adenocarcinoma was further supported with the negative results for PAX8, HNF-1 $\beta$, ER and PR, and history of lung adenocarcinoma.

Dominant oncogenes in NSCLC are associated with different biological behaviors manifesting as distinct patterns of metastasis, and $A L K$ rearrangement predisposes to rare sites of pericardial and pleural disease [31, 32]. Interestingly, in the present study, 11/16 (69\%) cases harbored $A L K$ rearrangements in an unselected rare ovarian metastasis from a lung adenocarcinoma as the most frequent molecular alteration. EML4-ALK fusion genes are predominantly observed in younger, nonsmoking/light smoking female populations, especially in East Asia. Histopathologically, this $A L K$ rearrangement in lung adenocarcinoma appears to promote the unique features of solid and/or signet ring cells [33]. Other features have been found in cases with $A L K$ rearrangements, whereby mixed subtype adenocarcinomas were the most common subtype, followed by the acinar predominant subtype, papillary predominant subtype [34], micropapillary and cribriform growth [35, 36], bronchioloalveolar carcinoma (BAC) and low-grade endobronchial mucoepidermoid carcinoma. Rare casesof adenosquamous carcinoma and mucoepidermoid carcinoma has been reported [35]. Although there is one reported case of ovarian metastatic lung adenocarcinoma with comprehensive solid and signet ring cells [16], regrettably, there was no further genetic analysis of this case. Our results also indicated that the solid phenotype is highly suggestive of $A L K$ alteration, and 4/5 cases with solid features harbored ALK rearrangement. However, the acinar pattern was slightly more common than was the solid pattern in patients with $A L K$ rearrangement ( 6 acinar vs. 5 solid), which is consistent with previous reports in which lung adenocarcinoma with $A L K$ rearrangement was likely to be related to acinar components [37].

Notably, lung cancer with $A L K$ rearrangement represents only a small subset of NSCLC, but metastatic ovarian tumors were found to occur in most $A L K$ fusionpositive cases. Why the ovary is the favored site of these lung cancers is not known. In our cohort, the ALK protein was expressed in 4 cases (57\%), and 100\% concordance with the FISH results was observed. Most evidence demonstrates good correlation between the immunohistochemical staining of ALK (clone D5F3) and FISH [38]. To reduce the economic burden, patients should be screened by $A L K$ immunohistochemistry to ensure whether they are suitable for targeted therapy. Notably, pleural metastatic tumors weakly express $A L K$ (clone D5F3), but $75 \%$ were positive for ALK rearrangement by FISH; therefore, the results of immunohistochemical and FISH may be inconsistent for distant metastases [38].

Recently, Gupta $\mathrm{R}$ et al. demonstrated a decreased median survival rate for $A L K$-positive tumors with uncommon sites of metastasis compared with common sites [31]. However, only one patient with ovarian metastatic $A L K$-positive tumors was reported in their paper (this was not reanalyzed in the current study due to lack of detail information). According to our data, slightly increased survival compared with triple-negative (no EGRF/KRAS/ALK alterations) and EGFR/KRAS mutation cases may be associated with uncommon metastatic ovary tumors with ALK rearrangement, though this difference was not statistically significant. As mentioned, patients with $A L K$ rearrangement have a better prognosis than do those with wild-type EGFR [39]. Hence, patients with $A L K$ rearrangement and uncommon sites of metastasis to the ovary may achieve better outcomes and longer survival times. Because of the paucity of ovarian metastasis from lung adenocarcinoma, longer follow-up times and more samples are needed for further research. 
The EGFR mutation is approximately $10-15 \%$, more frequent than $A L K$ rearrangement [1, 2]. Histologically, patients with $E G F R$ mutation more frequently display BAC or papillary components, but the incidence of solid features is lower than that in other patients [40, 41]. One patient in our group with an EGFR mutation in exon 19 (p.746-750del) showed the acinar subtype, which conformed to the morphological features, and the mutation was in concordance with that of the primary lung adenocarcinoma. According to previous reports, there is an approximate discordance rate of $16.2-27 \%$ between primary lung adenocarcinomas and corresponding metastases. One case showed L858R in a primary lung cancer but 2235-2249del in ovarian metastatic tumors [42]. Because the responsiveness to EGFR tyrosine kinase inhibitors tends to correlate with the EGFR mutation status in metastatic lesions compared with primary tumors [42, 43], metastatic tumors should be retested. Patients with rare EGFR mutations in metastatic ovarian carcinomas are likely to have a worse prognosis than are those with tumors with $A L K$ rearrangement.

\section{Conclusions}

In summary, our findings indicate that $A L K$ rearrangement is the most common molecular alteration in lung tumor metastasis to the ovary, followed by EGFR mutation. Although patients with ALK rearrangement appear to have a better prognosis than do those without ALK rearrangement, more cases with longer follow-up and multivariant analysis are needed to clarify this point.

\section{Abbreviations}

ALK: anaplastic lymphoma receptor tyrosine kinase; ARMS: amplification refractory mutation system; BAC: bronchioloalveolar carcinoma; NSCLC: Nonsmall-cell lung cancer

\section{Acknowledgements}

Not applicable.

\section{Authors' contributions}

RB and WTY designed the study and interpreted data. RB wrote the manuscript and WTY modified it. QMB, XLZ and XYZ analyzed FISH and Sanger Sequence results. $X C$ and $W H J$ performed the immunohistochemical staining. RB, XLX, SXT, YFC, HJG, XYT, BC, HLG diagnosed these rare cases. All authors read and approved the final manuscript.

\section{Funding}

This work was supported by grants from the National Natural Foundation Science of China (NSFC 81802597).

\section{Availability of data and materials}

All data generated or analyzed during this study are included in this published article.

\section{Ethics approval and consent to participate}

The present study was approved by Fudan university cancer center ethics committee and consented to participate.

\section{Consent for publication}

We confirm that the person's data in any form in this paper were approved by the person according to our consent form of Fudan university cancer center.

\section{Competing interests}

The authors declare that they have no competing interests.

Received: 9 May 2019 Accepted: 1 August 2019

Published online: 28 August 2019

\section{References}

1. Soda M, Choi $Y L$, Enomoto $M$, et al. Identification of the transforming EML4-ALK fusion gene in non-small-cell lung cancer. Nature. 2007;448: $561-6$.

2. Barlesi F, Mazieres J, Merlio JP, et al. Routine molecular profiling of patients with advanced non-small-cell lung cancer: results of a 1-year nationwide programme of the French cooperative thoracic intergroup (IFCT). Lancet. 2016;387:1415-26.

3. Gainor JF, Varghese AM, Ou SH, et al. ALK rearrangements are mutually exclusive with mutations in EGFR or KRAS: an analysis of 1,683 patients with non-small cell lung cancer. Clin Cancer Res. 2013;19:4273-81.

4. Zhang X, Zhang S, Yang X, et al. Fusion of EML4 and ALK is associated with development of lung adenocarcinomas lacking EGFR and KRAS mutations and is correlated with ALK expression. Mol Cancer. 2010:9:188.

5. Popat S, Vieira de Araujo A, Min T, et al. Lung adenocarcinoma with concurrent exon 19 EGFR mutation and ALK rearrangement responding to erlotinib. J Thorac Oncol. 2011;6:1962-3.

6. Zhou J, Zheng J, Zhao J, Sheng Y, Ding W. Poor response to gefitinib in lung adenocarcinoma with concomitant epidermal growth factor receptor mutation and anaplastic lymphoma kinase rearrangement. Thorac Cancer. 2015;6:216-9.

7. Mazur MT, Hsueh S, Gersell DJ. Metastases to the female genital tract Analysis of 325 cases. Cancer. 1984;53:1978-84

8. Irving JA, Young $\mathrm{RH}$. Lung carcinoma metastatic to the ovary: a clinicopathologic study of 32 cases emphasizing their morphologic spectrum and problems in differential diagnosis. Am J Surg Pathol. 2005;29: 997-1006.

9. Niu FY, Zhou Q, Yang JJ, et al. Distribution and prognosis of uncommon metastases from non-small cell lung cancer. BMC Cancer. 2016;16:149.

10. Lee KA, Lee JS, Min JK, et al. Bilateral ovarian metastases from ALK rearranged non-small cell lung Cancer. Tuberc Respir Dis (Seoul). 2014;77: 258-61.

11. Fujiwara A, Higashiyama M, Kanou T, et al. Bilateral ovarian metastasis of non-small cell lung cancer with ALK rearrangement. Lung Cancer. 2014;83: 302-4.

12. West $A H$, Yamada SD, MacMahon $H$, et al. Unique metastases of ALK mutated lung cancer activated to the adnexa of the uterus. Case Rep Clin Pathol. 2014;1:151-4.

13. Mushi RT, Yang Y, Cai Q, et al. Ovarian metastasis from non-small cell lung cancer with ALK and EGFR mutations: a report of two cases. Oncol Lett. 2016;12:4361-6.

14. Wang W, Wu W, Zhang Y. Response to crizotinib in a lung adenocarcinoma patient harboring EML4-ALK translocation with adnexal metastasis: a case report. Medicine (Baltimore). 2016;95:e4221.

15. Jing $X$, Li F, Meng $X$, et al. Ovarian metastasis from lung adenocarcinoma with ALK-positive rearrangement detected by next generation sequencing: a case report and literatures review. Cancer Biol Ther. 2017:18:279-84.

16. Giordano G, Cruz Viruel N, Silini EM, Nogales FF. Adenocarcinoma of the lung metastatic to the ovary with a signet ring cell component. Int J Surg Pathol. 2017;25:365-7.

17. Sasano $H$, Sekine A, Hirata $T$, et al. Ovarian metastases from ALK-rearranged lung adenocarcinoma: a case report and literature review. Intern Med. 2018; 57:3271-5.

18. Bi R, Bai QM, Yang F, et al. Microcystic stromal tumour of the ovary: frequent mutations of beta-catenin (CTNNB1) in six cases. Histopathology. 2015;67:872-9. 
19. Li Z, Dacic S, Pantanowitz L, et al. Correlation of cytomorphology and molecular findings in EGFR+, KRAS+, and ALK+ lung carcinomas. Am J Clin Pathol. 2014;141:420-8.

20. Trejo Bittar HE, Luvison A, Miller C, Dacic S. A comparison of ALK gene rearrangement and ALK protein expression in primary lung carcinoma and matched metastasis. Histopathology. 2017;71:269-77.

21. Paralkar VR, Li T, Langer CJ. Population characteristics and prognostic factors in metastatic non-small-cell lung cancer: a fox Chase Cancer Center retrospective. Clin Lung Cancer. 2008;9:116-21.

22. Quint LE, Tummala S, Brisson LJ, et al. Distribution of distant metastases from newly diagnosed non-small cell lung cancer. Ann Thorac Surg. 1996; 62:246-50.

23. Diem S, Fruh M, Rodriguez R, Liechti P, Rothermundt C. EML4-ALK-positive pulmonary adenocarcinoma with an unusual metastatic pattern: a case report. Case Rep Oncol. 2013;6:316-9.

24. Sooa Choi CKP, Kim SY, Yoon HK, Ro SM, Nam Y. Uterine cervix metastasis in lung adenocarcinoma with anaplastic lymphoma kinase rearrangement. Soonchunhyang Medical Science. 2015;21:142-5.

25. Chuang $X$, Chen Y, Yu $P$, et al. ALK rearrangement in lung adenocarcinoma with concurrent cervix and breast metastases: a case report. Thorac Cancer. 2018;9:1513-8.

26. Robert J. Kurman MLC, C. Simon Herrington, Robert H. Young. WHO Classification of tumors of female reproductive organs. International Agency for Research on Cancer, Lyon 2014; 4th edition, 27.

27. Lee $\mathrm{KR}$, Young $\mathrm{RH}$. The distinction between primary and metastatic mucinous carcinomas of the ovary: gross and histologic findings in 50 cases. Am J Surg Pathol. 2003;27:281-92.

28. Seidman JD, Kurman RJ, Ronnett BM. Primary and metastatic mucinous adenocarcinomas in the ovaries: incidence in routine practice with a new approach to improve intraoperative diagnosis. Am J Surg Pathol. 2003;27:985-93.

29. Ye J, Hameed O, Findeis-Hosey JJ, et al. Diagnostic utility of PAX8, TTF-1 and napsin a for discriminating metastatic carcinoma from primary adenocarcinoma of the lung. Biotech Histochem. 2012;87:30-4.

30. Ye J, Findeis-Hosey JJ, Yang Q, et al. Combination of napsin a and TTF-1 immunohistochemistry helps in differentiating primary lung adenocarcinoma from metastatic carcinoma in the lung. Appl Immunohistochem Mol Morphol. 2011:19:313-7.

31. Gupta R, Amanam I, Rahmanuddin S, et al. Anaplastic lymphoma kinase (ALK)-positive tumors: clinical, radiographic and molecular profiles, and uncommon sites of metastases in patients with lung adenocarcinoma. Am J Clin Onco. 2019;42:337-44

32. Doebele RC, Lu X, Sumey C, et al. Oncogene status predicts patterns of metastatic spread in treatment-naive nonsmall cell lung cancer. Cancer. 2012;118:4502-11.

33. Hou $L$, Ren $S$, Su B, et al. High concordance of ALK rearrangement between primary tumor and paired metastatic lymph node in patients with lung adenocarcinoma. J Thorac Dis. 2016;8:1103-11.

34. Takahashi T, Sonobe M, Kobayashi M, et al. Clinicopathologic features of non-small-cell lung cancer with EML4-ALK fusion gene. Ann Surg Oncol. 2010;17:889-97.

35. Wong DW, Leung EL, So KK, et al. The EML4-ALK fusion gene is involved in various histologic types of lung cancers from nonsmokers with wild-type EGFR and KRAS. Cancer. 2009;115:1723-33.

36. Kamata T, Yoshida A, Shiraishi K, et al. Mucinous micropapillary pattern in lung adenocarcinomas: a unique histology with genetic correlates. Histopathology. 2016;68:356-66.

37. Inamura K, Takeuchi K, Togashi Y, et al. EML4-ALK lung cancers are characterized by rare other mutations, a TTF-1 cell lineage, an acinar histology, and young onset. Mod Pathol. 2009;22:508-15.

38. Sun PL, Seol H, Lee HJ, et al. High incidence of EGFR mutations in Korean men smokers with no intratumoral heterogeneity of lung adenocarcinomas: correlation with histologic subtypes, EGFR/TTF-1 expressions, and clinical features. J Thorac Oncol. 2012;7:323-30.

39. Chen Z, Liu X, Zhao J, Yang H, Teng X. Correlation of EGFR mutation and histological subtype according to the IASLC/ATS/ERS classification of lung adenocarcinoma. Int J Clin Exp Pathol. 2014;7:8039-45.

40. Han HS, Eom DW, Kim JH, et al. EGFR mutation status in primary lung adenocarcinomas and corresponding metastatic lesions: discordance in pleural metastases. Clin Lung Cancer. 2011;12:380-6.
41. Gow CH, Chang YL, Hsu YC, et al. Comparison of epidermal growth factor receptor mutations between primary and corresponding metastatic tumors in tyrosine kinase inhibitor-naive non-small-cell lung cancer. Ann Oncol. 2009:20:696-702.

42. Demidova I, Barinov A, Savelov N, et al. Immunohistochemistry, fluorescence in situ hybridization, and reverse transcription-polymerase chain reaction for the detection of anaplastic lymphoma kinase gene rearrangements in patients with non-small cell lung cancer: potential advantages and methodologic pitfalls. Arch Pathol Lab Med. 2014;138:794-802.

43. Wu SG, Kuo YW, Chang YL, et al. EML4-ALK translocation predicts better outcome in lung adenocarcinoma patients with wild-type EGFR. J Thorac Oncol. 2012;7:98-104.

\section{Publisher's Note}

Springer Nature remains neutral with regard to jurisdictional claims in published maps and institutional affiliations.
Ready to submit your research? Choose BMC and benefit from:

- fast, convenient online submission

- thorough peer review by experienced researchers in your field

- rapid publication on acceptance

- support for research data, including large and complex data types

- gold Open Access which fosters wider collaboration and increased citations

- maximum visibility for your research: over $100 \mathrm{M}$ website views per year

At BMC, research is always in progress.

Learn more biomedcentral.com/submissions 